\title{
Early Detection of Multiorgan Light-Chain Amyloidosis by Whole-Body ${ }^{18}$ F-Florbetapir PET/CT
}

\author{
Eric C. Ehman ${ }^{1}$, M. Samir El-Sady ${ }^{1}$, Marie F. Kijewski ${ }^{1}$, Yiu Ming Khor ${ }^{1}$, Sophia Jacob ${ }^{1}$, Frederick L. Ruberg ${ }^{2}$, \\ Vaishali Sanchorawala ${ }^{2}$, Heather Landau ${ }^{3}$, Andrew J. Yee ${ }^{4}$, Giada Bianchi ${ }^{5}$, Marcelo F. Di Carli ${ }^{1,6}$, Rodney H. Falk ${ }^{7}$, \\ Hyewon Hyun ${ }^{1}$, and Sharmila Dorbala ${ }^{1,6,7}$ \\ ${ }^{1}$ Division of Nuclear Medicine, Department of Radiology, Brigham and Women's Hospital, Boston, Massachusetts; ${ }^{2}$ Amyloidosis \\ Center, Boston University School of Medicine, Boston, Massachusetts; ${ }^{3}$ Division of Medical Oncology, Memorial Sloan Kettering \\ Medical Center, New York, New York; ${ }^{4}$ Division of Hematology and Oncology, Department of Medicine, Massachusetts General \\ Hospital, Boston, Massachusetts; ${ }^{5}$ Division of Medical Oncology, Dana Farber Cancer Institute, Boston, Massachusetts; ${ }^{6} \mathrm{CV}$ \\ Imaging Program, Cardiovascular Division, Brigham and Women's Hospital, Boston, Massachusetts; and ${ }^{7}$ Cardiac Amyloidosis \\ Program, Division of Cardiology, Department of Medicine, Brigham and Women's Hospital, Boston, Massachusetts
}

\begin{abstract}
Immunoglobulin light-chain (AL) amyloidosis affects multiple systemic organs. However, determination of the precise extent of organ involvement remains challenging. Targeted amyloid imaging with ${ }^{18} \mathrm{~F}$-florbetapir PET/CT offers the potential to detect AL deposits in multiple organs. The primary aim of this study was to determine the distribution and frequency of $A L$ deposits in the various organs of subjects with systemic AL amyloidosis using ${ }^{18} \mathrm{~F}$-florbetapir PET/ CT. Methods: This prospective study included 40 subjects with biopsy-proven $\mathrm{AL}$ amyloidosis including active $\mathrm{AL}$ amyloidosis $(n=$ 30) or AL amyloidosis in hematologic remission for more than $1 \mathrm{y}$ $(n=10)$. All subjects underwent ${ }^{18} \mathrm{~F}$-florbetapir PET/CT, skull base to below the kidney scan field, from 60 to 90 min after injection of radiotracer. Volume-of-interest measurements of SUV $\max$ were obtained using Hermes software for the parotid gland, tongue, thyroid, lung, gastric wall, pancreas, spleen, kidney, muscle, abdominal fat, lower thoracic spine, vertebral body, and humeral head. Uptake in each organ was visually compared with that in spine bone marrow. An SUV max $_{\text {max }}$ of at least 2.5 was considered abnormal in all organs other than the liver. Results: Compared with the international consensus definition of organ involvement, ${ }^{18} \mathrm{~F}$-florbetapir $\mathrm{PET} / \mathrm{CT}$ identified amyloid deposits in substantially higher percentages of subjects for several organ systems, including parotid gland (50\% vs. $3 \%)$, tongue (53\% vs. $10 \%)$, and lung (35\% vs. $10 \%)$. In several organ systems, including kidney (13\% vs. $28 \%$ ) and abdominal wall fat (10\% vs. $13 \%)$, PET identified involvement in fewer subjects than did international consensus. Quantitative analysis of ${ }^{18} \mathrm{~F}$-florbetapir PET/CT revealed more frequent organ involvement than did visual analysis in the tongue, thyroid, lung, pancreas, kidney, muscle, and humeral head. Extensive organ amyloid deposits were observed in active $A L$ as well as in AL remission cohorts, and in both cardiac and noncardiac AL cohorts. Conclusion: ${ }^{18} \mathrm{~F}-$ florbetapir PET/CT detected widespread organ amyloid deposition in subjects with both active $A L$ and $A L$ hematologic remission. In most instances, amyloid deposits in the various organs were not associated with clinical symptoms and, thus, were unrecognized. Early recognition of systemic organ involvement may help tailor treatment,
\end{abstract}

Received Oct. 28, 2018; revision accepted Jan. 30, 2019.

For correspondence or reprints contact: Sharmila Dorbala, Brigham and Women's Hospital and Harvard Medical School, 75 Francis St., Boston, MA 02115.

E-mail: sdorbala@bwh.harvard.edu

Published online Apr. 6, 2019.

COPYRIGHT (C) 2019 by the Society of Nuclear Medicine and Molecular Imaging. and noninvasive monitoring of organ-level disease may guide management with novel fibril-resorbing therapies.

Key Words: systemic light chain amyloidosis; ${ }^{18} \mathrm{~F}$-florbetapir; PET/ CT; organ; AL

J Nucl Med 2019; 60:1234-1239

DOI: $10.2967 /$ jnumed.118.221770

I mmunoglobulin light-chain (AL) amyloidosis results from the deposition of immunoglobulin light chains produced by abnormal plasma cells as amyloid fibrils (1-3). It affects multiple organ systems, including the kidneys, heart, gastrointestinal tract, lung, nerves, and soft tissues, including the tongue, skin, muscles, and joints (4). At least 2 organ systems are involved in most AL subjects, resulting in substantial morbidity $(5,6)$; a greater number of organs involved may portend a dire prognosis. Cardiac involvement is the leading cause of mortality in subjects with systemic AL amyloidosis (7); however, whether this is a direct result of cardiac involvement or due to a greater organ burden of AL amyloidosis is not known.

Diagnostic methods for organ involvement in systemic AL amyloidosis are extremely limited. The international consensus criteria are a combination of clinical symptoms, serum cardiac biomarkers, liver function tests, urine protein levels, and available tissue biopsy (8). Overt clinical symptoms and laboratory features of organ dysfunction typically reflect advanced amyloidosis and, therefore, cannot detect early organ involvement before clinical manifestations.

Targeted whole-body imaging with amyloid molecular tracers offers the potential to detect amyloid deposits and, consequently, to identify the systemic organs involved in individuals with active $\mathrm{AL}$ or AL remission, even in the absence of organ-specific clinical symptoms. ${ }^{18} \mathrm{~F}$-florbetapir is one of a family of emerging PET radiopharmaceuticals that target $\beta$-amyloid deposits (9) and is Food and Drug Administration-approved for diagnosing Alzheimer disease. Recent in vivo (10) and ex vivo (11) studies have confirmed its utility in the evaluation of cardiac AL amyloidosis. Amyloid imaging using ${ }^{123} \mathrm{I}$ serum amyloid $\mathrm{P}$ component has been used, but this agent is limited by planar/SPECT imaging and is not currently available in the United States (12). 
The primary aim of this study was to determine whether AL deposits in the various organs can be identified using ${ }^{18} \mathrm{~F}$-florbetapir PET/CT imaging independent of clinical manifestations. We hypothesized that ${ }^{18} \mathrm{~F}$-florbetapir PET/CT imaging would identify amyloid deposits in more sites than are identified using the international consensus definition of organ involvement in AL amyloidosis. We also aimed to evaluate amyloid deposits in the various organs in subjects who achieved hematologic remission for more than 1 y compared with subjects with active AL, and in subjects with cardiac AL compared with those without cardiac AL amyloidosis.

\section{MATERIALS AND METHODS}

\section{Patient Selection}

We evaluated 40 subjects with biopsy-proven systemic AL amyloidosis enrolled in a prospective study, "Molecular Imaging of Primary Amyloid Cardiomyopathy" (ClinicalTrials.gov identifier NCT02641145). Inclusion and exclusion criteria for the study are listed in Supplemental Table 1 (supplemental materials are available at http://jnm.snmjournals. org). The study was approved by the institutional Human Research Committee. All subjects provided written informed consent for this study.

\section{${ }^{18}$ F-Florbetapir PET/CT Image Acquisition}

Subjects underwent PET/CT using a Discovery DRX imaging system (GE Healthcare). Starting at $60 \mathrm{~min}$ after injection of $355 \pm 53$ $\mathrm{MBq}$ of ${ }^{18} \mathrm{~F}$-florbetapir (PETNET), subjects were imaged for $30 \mathrm{~min}$ from the skull base to below the kidneys with 5 min per PET bed position. CT images were acquired for attenuation correction using a noncontrast technique (10-mA tube current, 140-kVP tube voltage, free tidal breathing). PET data were reconstructed using ordered-subsets expectation maximization with 2 iterations and 21 subsets. Axial, coronal, and sagittal CT and PET reconstructions were generated automatically by the scanner.

\section{${ }^{18}$ F-Florbetapir PET/CT Image Analysis}

Fused PET/CT images were viewed using a Hermes workstation (Hermes Medical Solutions). Imaging studies were reviewed visually together by 3 imaging physicians and were qualitatively and semiquantitatively analyzed by a board-certified radiologist. Images were qualitatively assessed for uptake in the following organs: parotid gland, tongue, thyroid, lung (upper, middle, and lower), stomach, pancreas, spleen, kidney, muscle, subcutaneous fat, and humeral head bone marrow. Each organ was assigned a visual score on a $0-2$ scale ( 0 , no visual uptake; 1 , uptake $\leq$ lower thoracic spine bone marrow; 2 , uptake $>$ thoracic spine bone marrow).

On a Hermes workstation, quantitative analysis was performed using a fixed-size volume of interest (2-3 cm in diameter, depending on organ). Care was taken to avoid including excretory structures (biliary system, proximal small bowel, and renal collecting system) in the volumes measured. SUVs were calculated for each volume of interest. An $\mathrm{SUV}_{\max }$ greater than or equal to 2.5 was considered abnormal in all organs other than the liver.

Retention index during dynamic-phase imaging was calculated for the gastric wall and lumen over the first 10-30 min of scan time by taking the $\mathrm{SUV}_{\text {mean }}$ in each dynamic frame divided by the integral of the blood pool ${ }^{18} \mathrm{~F}$-florbetapir time-activity curve at that point.

\section{Definition of Organ Involvement}

All subjects in this study had documented histologic proof of AL amyloidosis. We specifically evaluated involvement of the parotid gland, tongue, thyroid, lung (upper, middle, and lower), liver, pancreas, spleen, kidney, abdominal wall or paraspinal muscle, anterior or posterior abdominal fat, lower thoracic vertebral body, and humeral head using clinical criteria and ${ }^{18} \mathrm{~F}$-florbetapir PET/CT.
Detailed clinical information was prospectively collected by a review of systems. Laboratory data were collected, including serum free light-chain levels, estimated glomerular filtration rate, hemoglobin, cardiac biomarkers (troponin $\mathrm{T}, \mathrm{N}$-terminal pro-B-type natriuretic peptide), liver function tests, spot urine protein, and 24-h urine protein in subjects with renal involvement. By clinical criteria, involvement of the kidney and soft tissue in systemic AL amyloidosis was defined per the international consensus definitions $(8)$ or by clinical manifestations or biopsy. Involvement of the parotid gland, fat, and bone marrow was assessed by clinical findings or biopsy. Lung was deemed to be involved if the subject had a pleural effusion that was refractory to medical therapy and required the placement of a pleural catheter or biopsy.

On ${ }^{18} \mathrm{~F}$-florbetapir PET/CT, organ involvement was assessed both qualitatively and quantitatively.

The heart, liver, and nervous system were not evaluated in this analysis. Cardiac AL amyloidosis is specifically not addressed in this analysis as it comprised an inclusion criterion for study enrollment into 2 of the 3 arms. Because ${ }^{18} \mathrm{~F}$-florbetapir is excreted through the hepatobiliary system, liver involvement could not be accurately evaluated on imaging. Involvement of the nervous system was not assessed by ${ }^{18} \mathrm{~F}$-florbetapir PET/CT.

\section{Data Analysis}

Continuous variables are described as means with SDs or medians with interquartile ranges as appropriate. Discrete variables are described using percentages. A $P$ value of less than 0.05 was considered significant. Statistical analysis was performed using Excel (Microsoft) and SPSS (IBM).

\section{RESULTS}

\section{Demographics}

The baseline characteristics of the 40 subjects in this study are included in Table 1. Of these, 30 had active AL amyloidosis (with or without cardiac involvement) and 10 had AL amyloidosis in hematologic remission after treatment of plasma cell dyscrasia for at least $1 \mathrm{y}$ after treatment (AL remission). The heart was involved in 32 subjects: $22(45 \%)$ with active systemic AL amyloidosis and $10(25 \%)$ with a diagnosis of cardiac AL amyloidosis who were in hematologic remission. The number of subjects diagnosed via endomyocardial biopsy was $25 \%$, but histologic diagnosis was established by biopsy of other organs in the remaining $75 \%$ of subjects.

\section{Organ Involvement by Clinical and ${ }^{18} \mathrm{~F}-$ Florbetapir PET/CT}

On the basis of international consensus criteria, the specific organs involved with amyloidosis included kidneys (11, 27.5\%), liver $(2,5 \%)$, gastrointestinal tract $(1,2.5 \%)$, lung $(4,10 \%)$, and soft tissue $(6,15 \%)$.

By visual assessment of ${ }^{18} \mathrm{~F}$-florbetapir PET/CT, 33 of 40 subjects $(82 \%)$ had amyloid deposits in at least 1 organ, with 6 of 40 $(15 \%)$ having a solitary organ involved, 6 of $40(15 \%)$ having 2 organs, 5 of $40(13 \%)$ having 3 organs, 6 of 40 (15\%) having 4 organs, 4 of $40(10 \%)$ having 5 organs, 1 of $40(3 \%)$ having 6 organs, 3 of $40(8 \%)$ having 7 organs, and 2 of $40(5 \%)$ having 8 organs involved. Of note, heart and liver involvement were not assessed in this study as discussed in the Materials and Methods section.

Amyloid deposits in the various organs of the subjects using quantitative ${ }^{18} \mathrm{~F}$-florbetapir PET/CT imaging and organized by study group are shown in a heat map format (Supplemental Fig. 1). SUV measurements are shown in Figure 1, using an $\mathrm{SUV}_{\max }$ 
TABLE 1

Baseline Characteristics of Study Subjects

\begin{tabular}{|c|c|}
\hline Characteristic & Data \\
\hline Age (y) & $61 \pm 7$ \\
\hline Active AL, systemic cardiac & 22 \\
\hline Active AL, systemic noncardiac & 8 \\
\hline Remission AL, cardiac & 10 \\
\hline Height (m) & $1.70 \pm 0.11$ \\
\hline Weight (kg) & $77.0 \pm 14.7$ \\
\hline Body mass index $\left(\mathrm{kg} / \mathrm{m}^{2}\right)$ & $26.5 \pm 4.0$ \\
\hline $\mathrm{K}(\mathrm{mg} / \mathrm{L})^{\star}$ & $15.4(10.3-27.6)$ \\
\hline$\lambda(\mathrm{mg} / \mathrm{L})^{\star}$ & $114.4(35.1-306.7)$ \\
\hline $\mathrm{k} / \lambda$ ratio* & $0.19(0.04-0.46)$ \\
\hline eGFR $\left(\mathrm{mL} / \mathrm{min} / 1.73 \mathrm{~m}^{2}\right)$ & $57 \pm 16$ \\
\hline 24-h urine protein (g) & $0.9 \pm 2.2$ \\
\hline Alkaline phosphatase (U/L) & $96 \pm 55$ \\
\hline
\end{tabular}

${ }^{*}$ For patients with active $\mathrm{AL}$ amyloidosis.

eGFR = estimated glomerular filtration rate.

Qualitative data are expressed as numbers; continuous data are expressed as mean \pm SD or as median followed by interquartile range in parentheses.

threshold of at least 2.5: 37 of 40 subjects (93\%) had amyloid deposits in at least 1 organ and 33 of $40(83 \%)$ had 2 or more organs involved. Specifically, 1 organ was involved in 4 of 40 (10\%), 2 organs were involved in 6 of $40(15 \%), 3$ in 6 of 40 $(15 \%), 4$ in 6 of $40(15 \%), 5$ in 5 of $40(13 \%), 6$ in 6 of $40(15 \%)$, 7 in 1 of $40(3 \%)$ and 8 in 3 of $40(8 \%)$.

Gastric wall retention index was $0.025 \pm 0.017 / \mathrm{min}$, and splenic retention index was $0.041 \pm 0.035 / \mathrm{min}$.

\section{Organ Involvement by Whole-Body ${ }^{18}$ F-Florbetapir PET/CT Compared with Clinical Symptoms}

${ }^{18} \mathrm{~F}$-florbetapir PET/CT detected greater numbers of organs with amyloid deposition than did clinical symptoms or international consensus criteria (Table 2; Fig. 2). Clinical symptoms and radiologic features of involvement with amyloidosis in the fat, marrow, pancreas, and spleen are not well known. Radiologically, lung involvement has been described as pleural effusions and other nonspecific findings (13). In subjects with elevated $\mathrm{SUV}_{\max }$ in lung parenchyma, the most common CT finding was an associated pleural effusion, seen in 11 of 14 (79\%). Effusions ranged in size from trace and unilateral to large and bilateral and could not be distinguished from heart failure. Within the lung parenchyma, the most commonly associated finding was diffuse ground-glass opacity, which was seen in 5 of 14 (36\%). Two of 14 subjects (14\%) had no abnormal CT findings in the lungs despite markedly abnormal ${ }^{18} \mathrm{~F}$-florbetapir uptake. Figure 3 shows a published (14) image of distribution of ${ }^{18} \mathrm{~F}$ florbetapir in a healthy volunteer. Figure 4 shows representative images of amyloid involvement of the various organs visualized on ${ }^{18} \mathrm{~F}$-florbetapir PET in 2 subjects with active systemic AL.

\section{Organ Involvement Subgroup Comparisons}

$\mathrm{SUV}_{\text {max }}$ data for the involved organs stratified by active AL compared with AL remission are shown in Table 3. The mean $\mathrm{SUV}_{\text {max }}$ for kidney was higher in active AL than in remission
AL $(2.7 \pm 0.7$ vs. $1.7 \pm 0.3, P=0.0002)$. All other organs showed similar uptake of ${ }^{18} \mathrm{~F}$-florbetapir. Figure 5 shows an image of amyloid involvement in the various organs in a subject with AL remission.

$\mathrm{SUV}_{\text {max }}$ for the involved organs was similar in active AL with and without cardiac involvement (Supplemental Table 2).

However, using a quantitative $\mathrm{SUV}_{\max }$ threshold of 2.5 on ${ }^{18} \mathrm{~F}$ florbetapir PET, $81 \%$ of the subjects with cardiac involvement (active or remission AL) showed involvement of at least 2 organs other than the heart, compared with $75 \%$ of the subjects with systemic AL without cardiac involvement.

\section{DISCUSSION}

Accurate and early assessment of organ involvement remains an important unmet clinical need in the management of subjects with systemic AL amyloidosis. The current study addressed that gap in detection of organ involvement in AL amyloidosis by evaluating the added value of ${ }^{18} \mathrm{~F}$-florbetapir PET/CT imaging to clinical and international consensus criteria. This study also explored, for the first time, to our knowledge, organ amyloid deposits in patients with $\mathrm{AL}$ amyloidosis in complete hematologic remission for more than 1 y. This study yielded several novel findings. In many organs, quantitative ${ }^{18} \mathrm{~F}$-florbetapir PET/CT was more sensitive than visual analysis, and both visual and quantitative ${ }^{18} \mathrm{~F}$-florbetapir PET/CT identified more organ involvement than did clinical manifestations and the international consensus definition of organ involvement in systemic AL amyloidosis. Additionally, ${ }^{18} \mathrm{~F}$-florbetapir PET/ CT identified amyloid deposits in the organs of patients thought to be in hematologic remission for more than $1 \mathrm{y}$.

Precise identification of organ involvement in AL amyloidosis may have important prognostic and therapeutic implications. Currently, diagnosis of organ involvement in systemic AL amyloidosis is based on clinical and laboratory evaluation (8). In subjects with widespread systemic amyloidosis, abdominal fat-pad biopsy is often confirmatory (15) for AL amyloidosis. However, there is a high rate of false-negative results on fat-pad biopsy, particularly in subjects with single-organ involvement, and therefore targeted end organ biopsy is often needed (16). Small tissue samples limit the utility of biopsy since they may not be representative of the whole organ. Histologic sampling of multiple organs within a single patient is not practical.

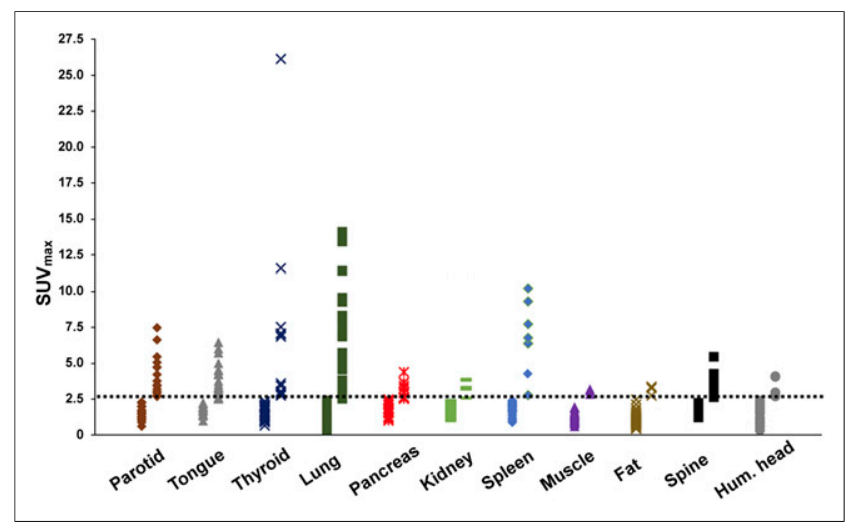

FIGURE 1. Detection of organ involvement by ${ }^{18} \mathrm{~F}$-florbetapir uptake. Distribution of SUV $V_{\max }$ values is shown for each organ. Each marker corresponds to single subject. Dotted line indicates assigned threshold of $2.5 \mathrm{SUV}_{\max }$ between normal and abnormal. Hum. = humeral. 
TABLE 2

Organ Involvement by Clinical Symptoms and by Visual and Quantitative Uptake of ${ }^{18} \mathrm{~F}$-Florbetapir in Various Organs in Systemic AL Amyloidosis

\begin{tabular}{lccccc}
\hline & & & & \multicolumn{2}{c}{ SUV max $_{\text {in subjects with... }}$} \\
\cline { 5 - 6 } Organ involved & Clinical diagnosis & Visual analysis & Quantitative analysis & Involved organs & Uninvolved organs \\
\hline Parotid & $1(3 \%)$ & $20(50 \%)$ & $19(48 \%)$ & $3.9 \pm 1.4$ & $1.5 \pm 0.5$ \\
\hline Tongue & $4(10 \%)$ & $17(43 \%)$ & $21(53 \%)$ & $3.9 \pm 1.1$ & $1.8 \pm 0.3$ \\
\hline Thyroid & - & $9(23 \%)$ & $14(36 \%)$ & $6.5 \pm 6.3$ & $1.5 \pm 0.5$ \\
\hline Lung & $4(10 \%)$ & $11(28 \%)$ & $14(35 \%)$ & $5.7 \pm 3.3$ & $1.0 \pm 0.6$ \\
Gastric wall & $1(3 \%)$ & $13(33 \%)$ & $15(38 \%)$ & $5.4 \pm 3.1$ & $1.8 \pm 0.4$ \\
Pancreas & - & $5(13 \%)$ & $16(40 \%)$ & $3.1 \pm 0.5$ & $1.9 \pm 0.5$ \\
Kidney & $11(28 \%)$ & $4(10 \%)$ & $5(13 \%)$ & $3.4 \pm 0.5$ & $1.9 \pm 0.3$ \\
\hline Spleen & - & $8(20 \%)$ & $8(20 \%)$ & $6.7 \pm 2.4$ & $1.8 \pm 0.4$ \\
Gluteal muscle & - & $1(3 \%)$ & $3(8 \%)$ & $3.1 \pm 0.2$ & $1.3 \pm 0.3$ \\
Abdominal wall fat & $5(13 \%)$ & $4(10 \%)$ & $3(8 \%)$ & $3.1 \pm 0.3$ & $1.1 \pm 0.4$ \\
\hline Humeral head & $8(20 \%)$ & $8(2 \%)$ & $6(15 \%)$ & $3.2 \pm 0.7$ & $1.4 \pm 0.6$
\end{tabular}

Qualitative data are expressed as numbers followed by percentages in parentheses; continuous data are expressed as mean \pm SD. Involved organs are defined as having a SUV $\max \geq 2.5$. Liver was considered unevaluable because of known hepatobiliary excretion of ${ }^{18} \mathrm{~F}$-florbetapir. Heart was not evaluated because it comprised study inclusion criteria. Some organs do not have consensus criteria for clinical diagnosis.

In the largest study of systemic AL amyloidosis to date, we evaluated the utility of ${ }^{18} \mathrm{~F}$-florbetapir PET/CT to map organ amyloid deposits. Our results indicate that whereas 27 of 40 subjects $(68 \%)$ had clinical manifestations of at least 1 organ other than the heart involved, partial whole-body ${ }^{18} \mathrm{~F}$-florbetapir PET/CT with quantitative imaging showed involvement by at least 1 organ in as many as 37 of 40 subjects (93\%). These findings suggest that, as with ${ }^{18} \mathrm{~F}$-FDG PET/CT for cancer imaging (17), systematic imaging-based disease staging using ${ }^{18} \mathrm{~F}$-florbetapir PET/CT is feasible

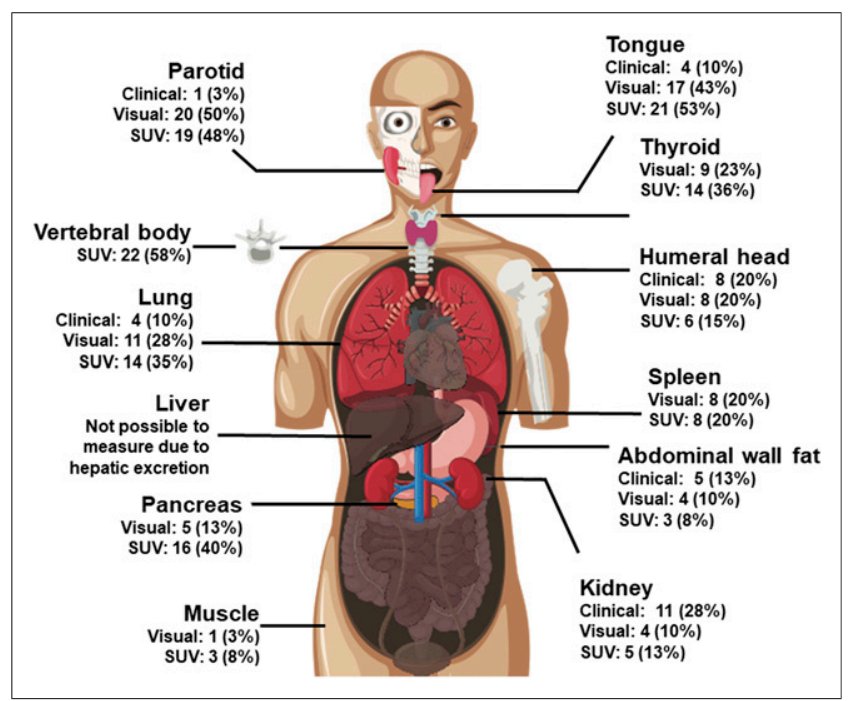

FIGURE 2. Proportion of subjects demonstrating ${ }^{18} \mathrm{~F}$-florbetapir uptake by organ. Visual involvement was defined as uptake greater than bone marrow. Quantitative assessment was deemed positive if SUV $\mathrm{max}_{\max }$ for volume of interest within organ was $\geq 2.5$. Vertebral body uptake was visual reference; therefore, subjective assessment of abnormal bone marrow uptake was not possible. and may provide incremental sensitivity to noninvasively identify disease burden even in the absence of symptoms or laboratory abnormalities. Involvement of the lungs, heart, soft tissue, and glands was well imaged by ${ }^{18} \mathrm{~F}$-florbepatir, whereas the liver and nervous system were unevaluable. Interestingly, the kidney, one of the most frequently involved organs in $\mathrm{AL}$ amyloidosis, was rarely determined by ${ }^{18} \mathrm{~F}$-florbetapir imaging to be involved. The precise reasons for this are not known and could be physiologic or technical. Patients with severe renal impairment (estimated glomerular filtration rate $<30 \mathrm{~mL} / \mathrm{min} / 1.73 \mathrm{~m}^{2}$ ) were excluded from enrollment, and it is possible this exclusion decreased the proportion of patients in our cohort with renal involvement. The tracer may not be able to access the amyloid fibrils in the kidneys, or the deposits may be below the imaging resolution, or the timing of imaging (60-90 min after injection of tracer) may not be optimized. Further study is warranted. Three smaller observational studies have explored whole-body radiotracer detection of amyloid fibrils in subjects with $\mathrm{AL}$ and transthyretin amyloidosis. One study used ${ }^{11} \mathrm{C}$-Pittsburgh compound $\mathrm{B}$ ( $n=7 \mathrm{AL}$ subjects) (18), another study used ${ }^{18} \mathrm{~F}$-florbetapir ( $n=15$ AL subjects) (19), and a final study used ${ }^{18} \mathrm{~F}$-florbetaben ( $n=7$ AL subjects) (20). Although direct comparison of proportions of involved organs is difficult given the small sample sizes in prior studies and the heterogeneity of organs examined, when overlapping organs studied in these papers were evaluated, the results among the 4 studies were similar. Compared with the other groups, we observed parotid uptake ( $48 \%$ vs. $57 \% / 40 \% / 57 \%$ ), tongue uptake (53\% vs. $100 \% / 43 \%$ ), thyroid uptake (36\% vs. $43 \% / 20 \% / 29 \%)$, kidney uptake (13\% vs. $7 \%$ / $29 \%$ ), muscle uptake ( $8 \%$ vs. $29 \%$ ), and lung uptake (35\% vs. $20 \%$ / $14 \%)$ at similar rates. In our study, spleen uptake (20\% vs. $57 \% / 40 \% / 43 \%$ ) and fat uptake ( $8 \%$ vs. $40 \%$ ) were seen less frequently. Our study adds to the results of the prior studies in a much larger cohort of subjects. Furthermore, our study described for the first 


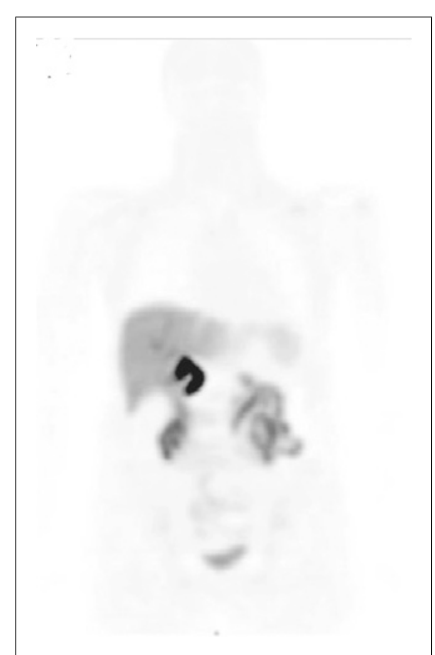

FIGURE 3. Normal ${ }^{18} \mathrm{~F}$-florbetapir biodistribution. PET maximum-intensity projection image shows ${ }^{18} \mathrm{~F}$-florbetapir distribution in healthy volunteer. (Reprinted with permission of (14).) opment of novel amyloid-resorbing drugs, to provide a baseline for evaluation of the effects from resorption of amyloid fibrils in the various organ systems.

Our study had several strengths and a few limitations. All subjects were prospectively enrolled using strict inclusion and exclusion criteria and were imaged for systemic amyloidosis. Images were interpreted in a masked fashion without knowledge of organ involvement or clinical symptoms. We included the full spectrum of amyloidosis, including active AL amyloidosis and AL remission. As the primary study was aimed at quantifying

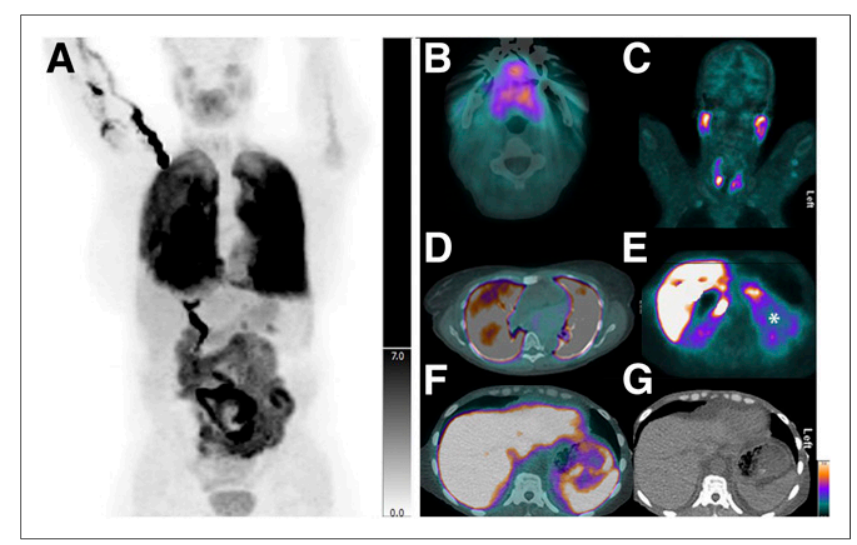

FIGURE 4. Images from 2 subjects with biopsy-proven active systemic $\mathrm{AL}$ amyloidosis. (A) Maximum-intensity projection image from ${ }^{18} \mathrm{~F}$-florbetapir PET/CT shows abnormal uptake in tongue and lungs. (B-D) Axial fused PET/CT images confirm diffuse abnormal uptake throughout tongue (B), parotids and thyroid (C), and both lungs (D). CT appearance of lungs was unremarkable other than mild atelectasis. (E-G) Abnormal uptake $\left(^{*}\right)$ is also seen in kidneys (E) and spleen ( $F$ and $G$ ).

cardiac amyloidosis, the dynamic images were obtained only through the level of the heart; therefore, retention index could not be calculated for many organs. The $\mathrm{SUV}_{\max }$ cutoff of at least 2.5 and bone marrow as the reference were chosen arbitrarily given the absence of a pool of known distribution in healthy volunteers. Quantitative reads were performed by a single reader, and results were not compared with a true reference standard; however, biopsy of all organs in all subjects is neither practical nor feasible. Future imaging of a large number of healthy volunteers could help better determine organ-specific physiologic $\mathrm{SUV}_{\max }$ thresholds. Because the radiotracer is excreted via the biliary system, evaluation of the liver, common bile duct, and small

TABLE 3

Organ Involvement in Subjects with Systemic AL Amyloidosis: Active AL $(n=30)$ Versus Hematologic Remission of $\mathrm{AL}(n=10)$

\begin{tabular}{|c|c|c|c|c|c|c|}
\hline \multirow[b]{2}{*}{ Organ } & \multicolumn{2}{|c|}{ Visual } & \multicolumn{2}{|c|}{ Quantitative } & \multicolumn{2}{|c|}{ SUV $_{\max }$} \\
\hline & Active & Remission & Active & Remission & Active & Remission \\
\hline Parotid & $15(50 \%)$ & $5(50 \%)$ & $14(47 \%)$ & $5(50 \%)$ & $2.7 \pm 1.6$ & $2.7 \pm 1.6$ \\
\hline Tongue & $13(43 \%)$ & $4(40 \%)$ & $15(50 \%)$ & $6(60 \%)$ & $2.9 \pm 1.3$ & $2.8 \pm 1.5$ \\
\hline Thyroid & $6(20 \%)$ & $3(30 \%)$ & $9(31 \%)$ & $5(50 \%)$ & $3.3 \pm 4.9$ & $3.0 \pm 2.3$ \\
\hline Lung & $9(30 \%)$ & $2(20 \%)$ & $11(37 \%)$ & $2(20 \%)$ & $2.2 \pm 2.8$ & $1.7 \pm 2.0$ \\
\hline Gastric wall & $13(43 \%)$ & $0(0 \%)$ & $14(47 \%)$ & $1(10 \%)$ & $3.5 \pm 2.9$ & $1.9 \pm 0.4$ \\
\hline Pancreas & $5(17 \%)$ & $0(0 \%)$ & $13(43 \%)$ & $3(30 \%)$ & $2.5 \pm 0.8$ & $2.1 \pm 0.7$ \\
\hline Kidney & $4(13 \%)$ & $0(0 \%)$ & $5(17 \%)$ & $0(0 \%)$ & $2.2 \pm 0.7^{*}$ & $1.7 \pm 0.3$ \\
\hline Spleen & $8(27 \%)$ & $0(0 \%)$ & 7 (23\%) & $1(10 \%)$ & $2.7 \pm 2.6$ & $1.5 \pm 1.7$ \\
\hline Gluteal muscle & $1(3 \%)$ & $0(0 \%)$ & $3(10 \%)$ & $0(0 \%)$ & $1.5 \pm 0.6$ & $1.3 \pm 0.2$ \\
\hline Abdominal wall fat & $4(13 \%)$ & $0(0 \%)$ & 3 (10\%) & $0(0 \%)$ & $1.2 \pm 0.8$ & $1.2 \pm 0.4$ \\
\hline Humeral head & $8(27 \%)$ & $0(0 \%)$ & $6(20 \%)$ & $0(0 \%)$ & $1.8 \pm 0.9$ & $1.2 \pm 0.5$ \\
\hline
\end{tabular}

${ }^{\star} P=0.0002$.

Qualitative data are expressed as numbers followed by percentages in parentheses; continuous data are expressed as mean \pm SD. Involved organs defined as SUV $\max \geq 2.5$. Liver was considered unevaluable because of known hepatobiliary excretion of ${ }^{18} \mathrm{~F}$-florbetapir. 


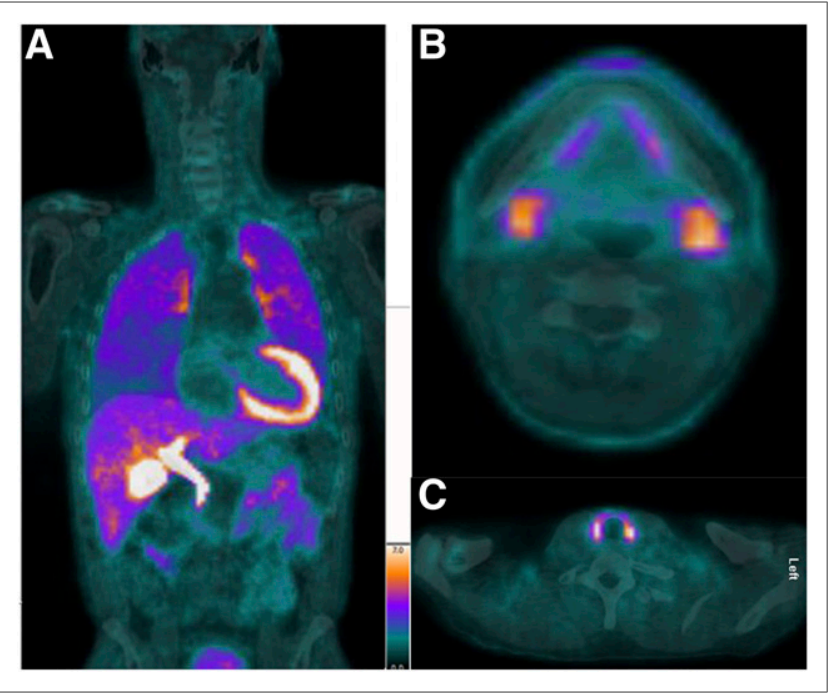

FIGURE 5. A 55-y-old man with cardiac $A L$ amyloidosis but in $A L$ remission for more than $1 \mathrm{y}$. (A) Coronal fusion ${ }^{18} \mathrm{~F}$-florbetapir PET/CT image shows abnormal uptake in lungs. (B and $C$ ) Axial fusion images show abnormal uptake in submandibular glands (B) and thyroid (C). This patient also had abnormal uptake in parotids and tongue (not pictured).

bowel was not possible as all subjects had varying levels of increased uptake in these organs.

\section{CONCLUSION}

${ }^{18} \mathrm{~F}$-florbetapir PET/CT is a promising technique for the evaluation of organ-level deposition of amyloid fibrils in systemic AL amyloidosis. In many cases, involved organs were not clinically evident. Imaging of organ involvement in systemic AL amyloidosis warrants further study because the ability to noninvasively diagnose organ-level disease may provide more accurate prognostication or identify candidates for the initiation of novel fibrilresorbing therapies. Finally, quantitation at the organ level holds promise for monitoring of organ response to effective plasma celldirected and novel antifibrillar therapies.

\section{DISCLOSURE}

Frederick Ruberg receives consulting fees from Pfizer, GlaxoSmithKline, and Caleum Biosciences and research support from Eidos Therapeutics. Vaishali Sanchorawala receives research support to the institution from Takeda, Celgene, Janssen, and Prothena and is on the scientific advisory board for Caleum Biosciences. Heather Landau receives consulting fees from Celgene, Takeda, Janssen, Prothena, Pfizer, and Juno and research support from Amgen, Spectrum, and Takeda. Marcelo Di Carli received a research grant from Spectrum Dynamics and Gilead and receives consulting fees from Sanofi and General Electric. Rodney Falk receives consulting fees from Ionis Pharmaceuticals and Alnylam Pharmaceuticals and research funding from GlaxoSmithKline. Sharmila Dorbala receives consulting fees from Pfizer, GE Healthcare, and AAA. Sharmila Dorbala and Rodney Falk are supported by an NIH RO1 grant (RO1 HL 130563), Sharmila Dorbala is supported by an American Heart Association grant (AHA 16 CSA 2888 0004), and Heather Landau is supported by an NIH/NCI Cancer Center grant (P30 CA008748). No other potential conflict of interest relevant to this article was reported.

\section{ACKNOWLEDGMENT}

We are extremely grateful to the study subjects and the study sites for their participation.

\section{KEY POINTS}

QUESTION: Can light-chain amyloid (AL) deposits in systemic organs be identified at ${ }^{18} \mathrm{~F}$-florbetapir PET/CT?

PERTINENT FINDINGS: In this prospective cohort study of subjects with biopsy-proven systemic AL amyloidosis, both quantitative and qualitative evaluation of ${ }^{18} \mathrm{~F}$-florbetapir PET/CT images identified $\mathrm{AL}$ deposits substantially more frequently than consensus guidelines based on clinical and laboratory manifestations.

IMPLICATIONS FOR PATIENT CARE: Organ-level detection of deposits may inform treatment and allow noninvasive monitoring of patients with $\mathrm{AL}$ amyloidosis.

\section{REFERENCES}

1. Bellotti V, Nuvolone M, Giorgetti S, et al. The workings of the amyloid diseases. Ann Med. 2007;39:200-207.

2. Glenner GG. Amyloid deposits and amyloidosis: the beta-fibrilloses (first of two parts). N Engl J Med. 1980;302:1283-1292.

3. Merlini G, Comenzo RL, Seldin DC, Wechalekar A, Gertz MA. Immunoglobulin light chain amyloidosis. Expert Rev Hematol. 2014;7:143-156.

4. Kyle RA, Gertz MA. Primary systemic amyloidosis: clinical and laboratory features in 474 cases. Semin Hematol. 1995;32:45-59.

5. Kumar SK, Gertz MA, Lacy MQ, et al. Recent improvements in survival in primary systemic amyloidosis and the importance of an early mortality risk score. Mayo Clin Proc. 2011;86:12-18.

6. Cibeira MT, Sanchorawala V, Seldin DC, et al. Outcome of AL amyloidosis after high-dose melphalan and autologous stem cell transplantation: long-term results in a series of 421 patients. Blood. 2011;118:4346-4352.

7. Falk RH, Alexander KM, Liao R, Dorbala S. AL (light-chain) cardiac amyloidosis: a review of diagnosis and therapy. J Am Coll Cardiol. 2016;68:1323-1341.

8. Gertz MA, Comenzo R, Falk RH, et al. Definition of organ involvement and treatment response in immunoglobulin light chain amyloidosis (AL): a consensus opinion from the 10th International Symposium on Amyloid and Amyloidosis, Tours, France, 18-22 April 2004. Am J Hematol. 2005;79:319-328.

9. Clark CM, Schneider JA, Bedell BJ, et al. Use of florbetapir-PET for imaging beta-amyloid pathology. JAMA. 2011;305:275-283.

10. Dorbala S, Vangala D, Semer J, et al. Imaging cardiac amyloidosis: a pilot study using ${ }^{18} \mathrm{~F}$-florbetapir positron emission tomography. Eur J Nucl Med Mol Imaging. 2014;41:1652-1662.

11. Park MA, Padera RF, Belanger A, et al. ${ }^{18} \mathrm{~F}$-florbetapir binds specifically to myocardial light chain and transthyretin amyloid deposits: autoradiography study. Circ Cardiovasc Imaging. 2015;8:e02954.

12. Hawkins PN, Lavender JP, Pepys MB. Evaluation of systemic amyloidosis by scintigraphy with ${ }^{123}$ I-labeled serum amyloid P component. $N$ Engl J Med. 1990;323:508-513.

13. Pickford HA, Swensen S, Utz J. Thoracic cross-sectional imaging of amyloidosis. AJR. 1997;168:351-355.

14. Joshi AD, Pontecorvo MJ, Adler L, et al. Radiation dosimetry of florbetapir F 18. EJNMMI Res. 2014;4:4.

15. Westermark P. Amyloid diagnosis, subcutaneous adipose tissue, immunohistochemistry and mass spectrometry. Amyloid. 2011;18:175-176.

16. Andrews TR, Colon-Otero G, Calamia KT, Menke DM, Boylan KB, Kyle RA. Utility of subcutaneous fat aspiration for diagnosing amyloidosis in patients with isolated peripheral neuropathy. Mayo Clin Proc. 2002;77:1287-1290.

17. Vansteenkiste JF, Stroobants SG, Leyn PRD, et al. Lymph node staging in nonsmall-cell lung cancer with FDG-PET scan: a prospective study on 690 lymph node stations from 68 patients. J Clin Oncol. 1998;16:2142-2149.

18. Ezawa N, Katoh N, Oguchi K, Yoshinaga T, Yazaki M, Sekijima Y. Visualization of multiple organ amyloid involvement in systemic amyloidosis using ${ }^{11} \mathrm{C}-\mathrm{PiB}$ PET imaging. Eur J Nucl Med Mol Imaging. 2018;45:452-461.

19. Wagner T, Page J, Burniston M, et al. Extracardiac ${ }^{18} \mathrm{~F}$-florbetapir imaging in patients with systemic amyloidosis: more than hearts and minds. Eur J Nucl Med Mol Imaging. 2018;45:1129-1138.

20. Baratto L, Park SY, Hatami N, et al. ${ }^{18} \mathrm{~F}$-florbetaben whole-body PET/MRI for evaluation of systemic amyloid deposition. EJNMMI Res. 2018;8:66. 